\title{
Inter- and Intra-Individual Variations of Heart Rate Variability in Japanese Males
}

\author{
Hiromitsu Kobayashi \\ Ishikawa Prefectural Nursing University
}

\begin{abstract}
Spectral components of heart rate variability (HRV) were repeatedly measured (4-8 measurements; mean: 6 measurements) in 75 healthy Japanese male subjects (age range: 20-61) under two postural conditions (standing and supine). Low-frequency (LF) and high-frequency (HF) components and mean heart rate (HR) were analyzed with special reference to individual variations, which were classified into two types: inter- (interV) and intra-individual variations (intraV). The percent contributions and coefficients of variations were calculated for both interV and intraV. Percent contributions of intraV (intraV\%) of $\mathrm{HR}$ with standing and supine postures were 15.8 and $12.9 \%$, respectively. The intra $\% \%$ of $\mathrm{HF}$ and LF on standing were 31.5 and $26.5 \%$, while those in the supine posture were 27.8 and $35.5 \%$, respectively. The coefficients of interV (interCV) and intraV (intraCV) were also calculated. The interCVs of HR, HF, and LF on standing were $14.9,41.4$, and $48.4 \%$, while those in the supine posture registered $16.2,42.9$, and $44.2 \%$, respectively. The intraCVs of HR, HF, and LF on standing were 5.0, 19.7, and $21.2 \%$, while those in the supine posture indicated 4.7 , 20.1 , and $23.0 \%$, respectively. We also calculated the interV and intraV of logarithmic-transformed HRV indexes. The logtransformation remarkably diminished both variables: interCV and intraCV registered 14-16 and 6-7\%, respectively. Although interV variations were considerabley large, intraV variations of HRV were negligibley small. The HRV indexes decreased with age, although HR remained unchanged. The coefficients of determination $\left(\mathrm{r}^{2}\right)$ were $14-34 \%$. In the case of log-transformed HRV indexes, the coefficients of determination registered $9-15 \%$, suggesting that $14-34 \%$ (raw) or $9-15 \%(\log )$ of the observed interV may be due to variations in age. J Physiol Anthropol 26(2): 173-177, 2007 http:// www.jstage.jst.go.jp/browse/jpa2

[DOI: 10.2114/jpa2.26.173]
\end{abstract}

Keywords: heart rate variability, inter-individual, intraindividual, reproducibility

\section{Introduction}

Human individual variation is one of the most important issues in the field of physiological anthropology. Individual variations may be classified into two types: inter- (interV) and intra-individual variations (intraV). These two variations have different perspectives; while interV represents the interaction between genotype and environment (including both natural and socio-cultural factors), intraV is a result of inconsistency of the physiological functions within a subject. Therefore, we need to discriminate the effects of interV from intra $\mathrm{V}$ in interpreting physiological indexes. If the measurements of an experiment were conducted only once, interV would not likely be discriminated from intraV. In short, we need to repeat multiple measurements of any physiological index in any one subject in an experiment to realize the discrepancies due to interV and intraV.

Heart rate variability (HRV), a non-invasive index of autonomic activity, has been employed in various fields of life sciences (Kobayashi, 1999). Although numerous studies on HRV have been attempted in recent years, this index occasionally produces controversial results due to the influence of individual variations. In ambulatory HRV related with monozygotic twins, genetic contributions of interV to SDNN and RMSSD (time-domain indexes) account for 35-47\% and $40-48 \%$, respectively (Kupper et al., 2004). With regard to high-frequency (HF) and low-frequency (LF) components, the genetic contributions of these frequency domain indexes are 13 and $16 \%$, respectively (Singh et al., 2001). A study on resting blood pressure has reported genetic contributions to systolic and diastolic blood pressures as 48-60 and 34-67\%, respectively (Hottenga et al., 2005). As such, the effect of environmental factors on individual differences of HRV is substantial when the blood pressure indexes are compared.

Concurrent investigations of interV and intraV of HRV are limited. According to a review study on the reliability and reproducibility of HRV by Sandercock et al. (2005), the extent of intraV influence on HRV indexes remains inconclusive. Therefore, we conducted repeated measurements of HRV in healthy Japanese males, and quantitatively examined the 
contributions of interV and intraV.

\section{Methods}

The heartbeat intervals in 75 healthy Japanese male subjects (age: 20-61 years) were monitored. In age distribution (Fig. 1), the measurements of the subjects were conducted in a series of two postural conditions: standing and supine. We repeatedly monitored the heartbeat intervals 2-4 times a day, and the same procedures were followed up 3 weeks after the first measurement. Therefore, measurements were repeated 4-8 times in total (mean frequency: 6 measurements). The order of experimental conditions between the experimental periods was changed to minimize the effect of circadian variation. After lunch at 11:00 hr, measurements were conducted on subjects between 13:00-16:00 hr. Respiration control was not applied in this study.

A wristwatch-type heart rate monitor (Polar S801i; Finland) was used for monitoring the heartbeat intervals, which were recorded for $4 \mathrm{~min}$ at a time-resolution of $1 \mathrm{msec}$. The heartbeat data (including beat-detection errors) were either corrected or excluded from analysis.

The sequences of heartbeat intervals were converted into beats per $\min (\mathrm{bpm})$, and interpolated as $5-\mathrm{Hz}$ equidistant data according to the instantaneous heart rate (HR) method described by De Boer et al. (1985).

Power spectra of HRV were calculated from 1024 points $(204.8 \mathrm{~s})$ of the interpolated heart rate sequences using fast Fourier transformation (FFT) processing. $\mathrm{HF}$ and LF components were integrations of the power spectra at their respective ranges of $0.15-0.35$ and $0.04-0.15 \mathrm{~Hz}$. In addition, the natural logarithmic (log)-transformed HRV indexes were calculated for comparison with the results of previous studies. In this case, we calculated the power spectrum based on the beat-to-beat interval sequence instead of instantaneous HR.

Using one-way repeated ANOVA, we calculated percent contributions of the interV (interV\%) and intraV (intraV\%).

inter $\mathrm{V} \%$ and intraV\% are defined by the following formulas:

$$
\text { inter } \%=S^{\prime} S_{b} / S_{t} \times 100
$$

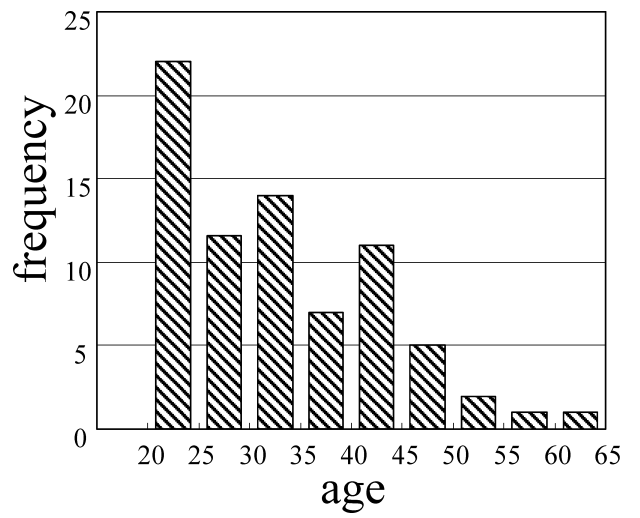

Fig. 1 Age distribution of subjects who participated in the study.

$$
\begin{aligned}
& \text { intraV } \%=100-\text { inter } V \% \\
& S_{b}^{\prime}=S_{b}-d f_{b} \times V_{e} \\
& S_{t}=S_{b}+S_{e}
\end{aligned}
$$

where

$S_{b}$ : sum-of-squares of interV

$S_{b}^{\prime}$ : pure sum-of-squares of interV

$S_{e}$ : sum-of-squares of intraV

$S_{t}$ : total sum-of-squares of interV and intraV

$d f_{b}$ : degree of freedom of interV

$V_{e}$ : variance of interV

We also calculated the coefficients of interV (interCV) and intraV (intraCV) to examine the extent of variations. Coefficients interCV and intraCV were derived from the following formula:

$$
\begin{aligned}
& \text { inter } C V=100 \frac{\sigma_{b}}{\bar{x}} \\
& \text { inter } C V=\frac{1}{n} \sum_{i}^{n} 100 \frac{\sigma_{e i}}{\bar{x}_{i}}
\end{aligned}
$$

where

$$
\begin{gathered}
\sigma_{b}=\left[\frac{1}{n} \sum_{i}^{n}\left(\bar{x}_{i}-\bar{x}\right)^{2}\right]^{\frac{1}{2}} \\
\sigma_{e i}=\left[\frac{1}{r} \sum_{j}^{r}\left(x_{i j}-\bar{x}_{i}\right)^{2}\right]^{\frac{1}{2}}
\end{gathered}
$$

where $n$ and $r$ are the number of subjects and repeats, respectively

\section{Results}

The results of the HR, HF, and LF (Fig. 2) revealed that when repeated measurements in a subject were plotted in a vertical line, the horizontal and vertical variations would respectively represent the interV and intraV. The distribution characteristics were different between the HR and HRV indexes. In the latter, the intra $\mathrm{V}$ correlated positively with the individual mean. In other words, a subject with a large HRV tended to show a large intraV. However, this tendency was not observed in HR.

According to the values of interV and intraV in the $\mathrm{HR}$ and HRV indexes (Table 1), the intraV\% of HR indicated 15.8 and $12.9 \%$ with the standing and supine postures, respectively. The HRV indexes showed a larger intraV than that of HR: i.e., approximating 30\%.

In the LF component, the intraV\% in the supine posture was larger than that on standing; however, HF showed a smaller 

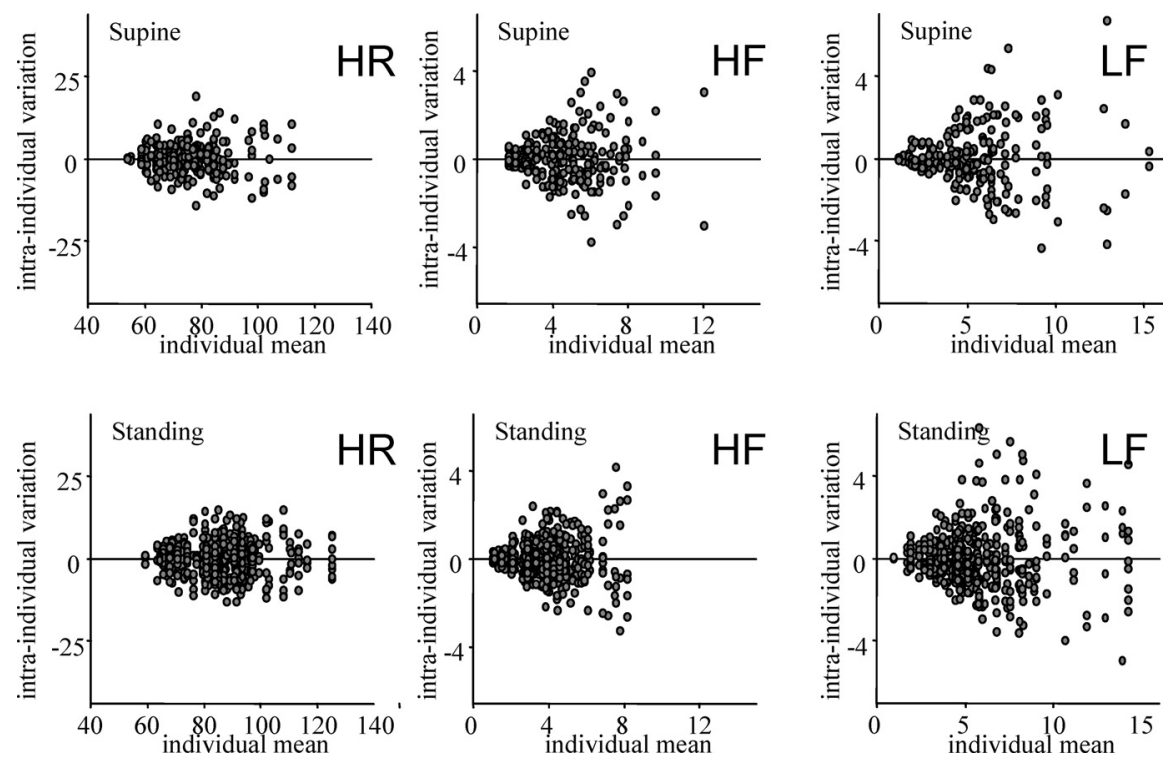

Fig. 2 Variations of the heart rate (HR), high-frequency (HF) and low-frequency (LF) components of HR variability (HRV). Each abscissa represents the mean value of intra-individual variations (intraV) for a subject, while the ordinate represents the mean of individual variations in a subject.

Table 1 Inter- (interV) and intra-individual variations (intraV) of heart rate (HR) and HR variability (HRV) indexes, such as high-frequency (HF) and low-frequency (LF) components. interV\% and intraV\% are percent contribution of inter- and intra-individual variations, respectively. Coefficients of interV and intraV are represented by interCV and intraCV, respectively

\begin{tabular}{rlrccccr}
\hline & posture & mean & $\mathrm{n}$ & interV\% & intraV\% & interCV (\%) & intraCV (\%) \\
\hline \multirow{2}{*}{ HR } & Standing & 86.33 & 450 & 84.2 & 15.8 & 14.9 & 5.0 \\
& Supine & 68.73 & 443 & 87.1 & 12.9 & 16.2 & 4.7 \\
HF & Standing & 3.89 & 450 & 68.5 & 31.5 & 41.4 & 19.5 \\
& Supine & 4.30 & 443 & 72.2 & 27.8 & 42.9 & 20.1 \\
& Standing & 6.26 & 450 & 73.5 & 26.5 & 48.4 & 21.2 \\
& Supine & 4.75 & 443 & 64.5 & 35.5 & 44.2 & 23.0 \\
\hline
\end{tabular}

Table 2 Inter- (interV) and intra-individual variations (intraV) of log-transformed HRV indexes, such as $\ln \mathrm{HF}$ and $\ln$ LF components

\begin{tabular}{clcccccc}
\hline & posture & mean & $\mathrm{n}$ & interV\% & intraV\% & interCV (\%) & intraCV (\%) \\
\hline \multirow{2}{*}{$1 \mathrm{nHF}$} & Standing & 3.35 & 450 & 78.7 & 21.3 & 16.2 & 7.2 \\
& Supine & 3.88 & 443 & 79.4 & 20.6 & 15.0 & 6.4 \\
\multirow{3}{*}{$\operatorname{lnLF}$} & Standing & 3.82 & 450 & 80.3 & 19.7 & 14.7 & 6.1 \\
& Supine & 3.98 & 443 & 76.5 & 23.5 & 13.5 & 5.9 \\
\hline
\end{tabular}

intraV\% in the supine posture. Because LF and HF indicated opposite responses to postural changes (Kobayashi, 1996), the above results are considered rational.

The coefficients of interV and intraV (interCV and intraCV) of HR approximated $15-16$ and 5\%, while those of HRV approached $41-48$ and $20 \%$, respectively (Table 1). Based on the contributions and CVs of log-transformed HRV indexes (Table 2), interCV and intraCV approximated to 15 and $6-7 \%$, respectively. The log-transformation remarkably diminished both interCV and intraCV.

The above intraV included both day-to-day and within-a-day variations. To examine the effect of time-span in repeated measurements, we calculated the intraV of data that included day-to-day variations and those of data that incorporated only within-a-day variations. In this calculation, the degree of freedom for each variance was 61 because of missing values. As the range of the variance ratio was 1.17-1.35 the difference of these two variances was considered insignificant. 
Table 3 Correlations of heart rate (HR) and HR variability (HRV) indexes with age. HRV indexes included high-frequency (HF) and low-frequency (LF) components. Each ordinate represents the individual mean of the index. The coefficient of determination is represented by $r^{2}$.

\begin{tabular}{lllrr}
\hline & posture & \multicolumn{1}{c}{ equation } & $r^{2}$ & $r^{2}(\%)$ \\
\hline \multirow{2}{*}{ HR vs age } & standing & $y=-0.44 x+102.22$ & -0.29 & 8 \\
& supine & $y=-0.10 x+72.06$ & -0.08 & 1 \\
HF vs age & standing & $y=-0.09 x+6.99$ & -0.58 & 34 \\
& supine & $y=-0.10 x+7.42$ & -0.50 & 25 \\
LF vs age & standing & $y=-0.14 x+10.03$ & -0.50 & 25 \\
& supine & $y=-0.08 x+6.85$ & -0.38 & 14 \\
lnHF vs age & standing & $y=-0.02 x+3.93$ & -0.33 & 11 \\
& supine & $y=-0.02 x+4.62$ & -0.39 & 15 \\
lnLF vs age & standing & $y=-0.02 x+4.38$ & -0.30 & 9 \\
& supine & $y=-0.02 x+4.56$ & -0.34 & 12 \\
\hline
\end{tabular}

The correlations of the individual means of HR and HRV with age (Table 3) indicated that HRV decreased with age, although such was not the case for HR. The log-transformed HRV indexes showed lower correlation coefficients than the results of non-transformed HRV indexes.

\section{Discussion}

\section{Ratio and magnitude of inter $V$ and intraV}

Although the intraV\% was $13-16 \%$ in $\mathrm{HR}$, LF and HF showed a larger intraV, i.e., approximately $30 \%$. It is of interest to note the results of Tango (1982) in various hematological and biochemical indexes; viz., the intraV\% of the number of red blood cells (RBC) and uric acid (SUA) concentration registered ca. 15 and $30 \%$, respectively. Therefore, the structures of individual variations in HR and HRV may respectively be similar to those of RBC and SUA.

In addition to the percent contribution, we examined the magnitude of variations by calculating interCV and intraCV, which approximated $15-16$ and 3\% for HR, respectively. In previous studies, the interCV of $\mathrm{HR}$ registered $15.6 \%$ in 32 males aged $48.9 \pm 6.8$ (Sinnreich et al., 1998), 10.9-12.6\% in 10 males and 10 females aged 28 2 (Pitzalis et al., 1996),

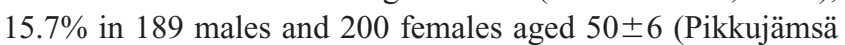
et al., 2001), and $16.5 \%$ in 472 males aged $40-79$ years (Kuo et al., 1999). Note that some of the above results on CV were based on the $\mathrm{R}-\mathrm{R}$ interval $(\mathrm{msec})$, whereas our results were based on the mean HR (bpm).

In the above studies, the interCV of log-transformed $\mathrm{HF}$ and LF at supine respectively registered $10.6-16.8 \%$ and $13.7-$ $17.6 \%$ (Pitzalis et al., 1996), and $18.5 \%$ and $13.2 \%$ (Pikkujämsä et al., 2001). The interCV of log-transformed HF and LF of this study were 15.0 and $13.5 \%$ at supine. Although age variations were not identical, most of the above studies have shown the interCV to display values almost similar to our findings.

In our results, the intraCV of log-transformed HRV approximated 6-7\%. When Sinnreich et al. (1998) measured the HRV of 70 normal subjects ( 32 males and 38 females from a kibbutz in Israel) in a supine position, the intraCV values of log-transformed $\mathrm{HF}$ and $\mathrm{LF}$ registered 12.1 and $11.5 \%$, respectively. However, the respective intraCV values of $\mathrm{HF}$ and LF were 28 and $27 \%$ in 14 normal subjects ( 5 females and 9 males) measured by Højgaard et al. (2005). The intraCV of our study indicated values smaller than those of previous studies.

From our results, the intraV values of HRV were negligibley small, although those for interV were considerabley large. Sandercock et al. (2005) have reviewed a number of studies on the reliability and reproducibility of HRV and concluded that the HRV indexes are reliable and reproducible. In short, our results are consistent with their conclusion.

\section{Effect of age on interV}

It is well known that the HRV indexes are affected by age, and the elderly tend to show a smaller HRV than the young (Yeragani et al., 1997; Kuo et al., 1999; Ziegler et al., 1999; Agelink et al., 2001). Our results also showed a decreased tendency in HRV analogous to previous results. In this study, the age of subjects ranged from 20 to 61 years, and this could have been a factor affecting the interV. The HRV indexes showed decreased tendencies with increasing age (Table 2). The coefficients of determination $\left(\mathrm{r}^{2}\right)$ were $11-34 \%$. In short, $11-34 \%$ of the observed interV might be due to age variation. The log-transformed indexes showed weaker correlations: the coefficients of determination were $9-15 \%$.

\section{Effect of posture on inter $V$ and intraV}

The present study investigated the effects of postural disposition on the intraV and interV of HRV. According to Nishikino et al. (2006), orthostatic changes in HRV components were influenced by single-nucleotide polymorphism (SNP), which is related to the renninangiotensin system (RAS). In other words, HRV specificities in individual differences are probably influenced by posture. Results in the present study revealed that the effects of posture on individual differences were more apparent in LF than HF components. Moreover, RAS influences the LF component of HRV via blood pressure variations (Akselrod et al., 1985). As such, our findings are probably most closely related with the results of Nishikino et al. (2006).

\section{Effect of diurnal variation on intraV}

A factor that possibly affects intraV is diurnal variation of HRV. Huikuri et al. (1994) have reported that maximal HF and minimal LF effects are observed early in the morning; however, the changes of HRV indexes during daytime were unclear. In our experiment, as measurements were conducted for $3-4 \mathrm{hr}$ in the afternoon, the effect of diurnal variation was considered insignificant.

Strictly speaking, intraV explored in this study incorporated both day-to-day and within-a-day variations, and differences in these two variations were not significant. Similar results have 
been reported by Kowalewski and Urban (2004); viz., the reproducibility of HRV yields no differences between within-aday and day-to-day (6 months) variations.

However, because of limitations on the experimental design, examination of this issue might be insufficient in this study. Further studies on the effects of time-span on intraV are warranted.

Acknowledgement This research was supported by a Grant-in-Aid for Scientific Research (B) (grant no. 15370104) from the Japanese Ministry of Education, Culture, Sports, Science and Technology.

\section{References}

Agelink MW, Malessa T, Baumann B, Majewski T, Akila F, Zeit T, Ziegler D (2001) Standardized tests of heart rate variability: normal ranges obtained from 309 healthy humans, and effects of age gender, and heart rate. Clinic Auton Res 11: 99-108

Akselrod S, Gordon D, Madwed JB, Snidman NC, Shannon DC, Cohen RJ (1985) Hemodynamic regulation: investigation by spectral analysis. Am J Physiol Heart Circ Physiol 249: H867-H875

De Boer RW, Karemaker JM, Strackee J (1985) Description of heart-rate variability data in accordance with a physiological model for the genesis of heartbeats. Psychophysiology 22(2): 147-154.

Huikuri HV, Niemelä MJ, Ojala S, Rantala A, Ikäheimo MJ, Airaksinen KE (1994) Circadian rhythms of frequency domain measures of heart rate variability in healthy subjects and patients with coronary artery disease. Effects of arousal and upright posture. Circulation 90: 121-126

Hottenga JJ, Boomsma DI, Kupper N, Posthuma D, Snieder H, Willemsen G, de Geus EJ (2005) Heritability and stability of resting blood pressure. Twin Res Hum Genet 8(5): 499-508

Højgaard MV, Holstein-Rathlou NH, Agner E, Kanters JK (2005) Reproducibility of heart rate variability, blood pressure variability and baroreceptor sensitivity during rest and head-up tilt. Blood Press Monit 10(1): 19-24

Kobayashi H (1996) Postural Effect on Respiratory Sinus Arrhythmia with Various Respiratory Frequencies. Appl Human Sci 15(2): 87-91.

Kobayashi H, Ishibashi K, Noguchi H (1999) Heart rate variability; an index for monitoring and analyzing human autonomic activities. Appl Human Sci 18(2): 53-59

Kowalewski MA, Urban M (2004) Short- and long-term reproducibility of autonomic measures in supine and standing positions. Clin Sci (Lond) 106: 61-66

Kuo TB, Lin T, Yang CC, Li CL, Chen CF, Chou P (1999) Effect of aging on gender differences in neural control of heart rate. Am J Physiol Heart Circ Physiol 277: H2233H2239
Kupper NH, Willemsen G, van den Berg M, de Boer D, Posthuma D, Boomsma DI, de Geus EJ (2004) Heritability of ambulatory heart rate variability. Circulation 110(18): 2792-2796

Nishikino M, Matsunaga T, Yasuda K, Adachi T, Moritani T, Tsujimoto G, Tsuda K, Aoki N (2006) Genetic variation in the renin-angiotensin system and autonomic nervous system function in young healthy Japanese subjects. J Clin Endocrin Metab 91: 4676-4681

Pikkujämsä SM, Makikallio TH, Airaksinen KE, Huikuri HV (2001) Determinants and interindividual variation of $\mathrm{R}-\mathrm{R}$ interval dynamics in healthy middle-aged subjects. Am J Physiol Heart Circ Physiol 280(3): H1400-H1406

Pitzalis MV, Mastropasqua F, Massari F, Forleo C, Di Maggio M, Passantino A, Colombo R, Di Biase M, Rizzon P (1996) Short- and long-term reproducibility of time and frequency domain heart rate variability measurements in normal subjects. Cardiovasc Res 32(2): 226-233

Sandercock GRH, Bromley PD, Brodi DA (2005) The reliability of short-term measurements of heart rate Variability. Int J Cardiol 103: 238-247

Singh JP, Larson MG, O’Donnell CJ, Levy D (2001) Genetic factors contribute to the variance in frequency domain measures of heart rate variability. Auton Neurosci 90(1-2): $122-126$

Sinnreich R, Kark JD, Friedlander Y, Sapoznikov D, Luria MH (1998) Five minute recording of heart rate variability for population studies: repeatability and age-sex characteristics. Heart 80: 156-162

Tango T (1982) An interpretation of normal ranges bases on individual difference quotient: extension to multivariate case. Med Inform (Lond) 7(2): 119-126

Yeragani VK, Sobolewski E, Kay J, Jampala VC, Igel G (1997) Effect of age on long-term heart rate variability. Cardiovasc Res 35: 35-42

Ziegler D, Srasburger K, Lambeck H, Dannehl K (1999) Normal ranges and reproducibility of statistical, geometric, frequency domain, and non-linear measures of 24-hour heart rate variability. Horm Metab Res 31: 672-679

This article was presented at the 8th International Congress of Physiological Anthropology, 2006 (ICPA 2006), in Kamakura, Japan

Received: September 29, 2006

Accepted: November 22, 2006

Correspondence to: Hiromitsu Kobayashi, Ishikawa Prefectural Nursing University, Nakanuma 7-1, Kahoku-city, Ishikawa 929-1212 Japan

Phone: +81-76-281-8344

Fax: +81-76-281-8356

e-mail: kobayasi@ishikawa-nu.ac.jp 